\title{
Pressure-induced Jahn-Teller suppression and simultaneous high-spin to low-spin transition in the layered perovskite $\mathrm{CsMnF}_{4}$
}

\author{
Fernando Aguado, ${ }^{1}$ Fernando Rodriguez, ${ }^{1, *}$ and Pedro Núñez ${ }^{2}$ \\ ${ }^{1}$ DCITIMAC, Facultad de Ciencias, Universidad de Cantabria, Santander 39005, Spain \\ ${ }^{2}$ Departamento de Química Inorgánica, Universidad de La Laguna, Tenerife 38200, Spain \\ (Received 16 May 2007; revised manuscript received 20 July 2007; published 25 September 2007)
}

\begin{abstract}
The interplay between the orbital ordering and the spin state in Jahn-Teller $\mathrm{Mn}^{3+}$ governing the optical, magnetic, and transport properties in the layered $\mathrm{CsMnF}_{4}$ perovskite is investigated. Such electronic effects are strongly coupled to the lattice and thus can be modified by external pressure. However, there is very little understanding of the structural conditions which are required to attain spin crossover in connection with the electronic structure of $\mathrm{Mn}^{3+}$. The distortion, spin state, and tilting of $\left(\mathrm{MnF}_{6}\right)^{3-}$ octahedra in the insulating ferromagnet $\mathrm{CsMnF}_{4}$ are jointly studied by high-pressure optical spectroscopy. The insulating character of $\mathrm{CsMnF}_{4}$ allowed us to explore the electronic structure associated with the $3 d$ levels of $\mathrm{Mn}^{3+}$ in the $0-46 \mathrm{GPa}$ pressure range, an information which is obscured in most oxides due to metallization at high pressure. We show that the spin-crossover transition, related to the spin change, $S=2 \rightarrow 1$, in $\mathrm{Mn}^{3+}$, takes place at $37 \mathrm{GPa}$ with the simultaneous suppression of the axially elongated distortion associated with the Jahn-Teller effect. The wide stability pressure range of the Jahn-Teller distortion and high-spin state is explained in terms of crystalfield models including the Jahn-Teller stabilization energy. On this basis, we discuss the interplay between spin transition and Jahn-Teller effect comparing present findings with other results attained in $\mathrm{Mn}^{3+}, \mathrm{Ni}^{3+}$, and $\mathrm{Co}^{3+}$ systems.
\end{abstract}

DOI: 10.1103/PhysRevB.76.094417

PACS number(s): 75.30.Wx, 71.70.Ch, 71.70.Ej, 81.40.Vw

\section{INTRODUCTION}

The magnetic moment of transition-metal ions can be abruptly reduced upon compression as a result of the increase in electronic energy dispersion caused by the electronelectron and electron-lattice interactions. ${ }^{1}$ For octahedral (O) transition-metal ions of a given electronic configuration $d^{n}$ $(4 \leqslant n \leqslant 5)$, the transition from high-spin $(S=n / 2)$ to lowspin $[S=(6-n) / 2]$, or from $S=(n-5) / 2$ to $S=(n-6) / 2$ if $(6 \leqslant n \leqslant 8)$, is governed by the competition between the crystal field, which splits the $3 d$ levels into a low-lying $t_{2}$ triplet and an excited $e$ doublet in $\Delta$, and the interelectronic repulsion and exchange energies for pairwise occupation of $d$ orbitals, $U$ (Fig. 1). If $\Delta>U$, the low-spin configuration is more favorable than the high-spin configuration. Strictly speaking, the spin transition in condensed matter systems will occur if the low-spin free energy is smaller than the high-spin free energy. Spin-transition phenomena have been intensively investigated in materials providing ligand fields close to the spin crossover. ${ }^{1-9}$ Current research of this kind is focused on materials involving transition-metal complexes with $\mathrm{C}, \mathrm{O}$, or $\mathrm{N}$ ligands. Their strong neuphelaxetic effect makes those complexes well suited to exhibiting bistability around ambient conditions. ${ }^{1}$ In general, transition-metal halides and oxides at ambient pressure exhibit high-spin magnetic moments as $\Delta$ is usually smaller than the crystal-field energy for spin crossover, $\Delta_{\mathrm{SCO}} \cdot{ }^{2-4}$

Similar to Earth's interior conditions, high-pressure techniques have emerged as efficient tools to attain structural conditions for spin change, even dealing with systems where $\Delta \ll \Delta_{\text {SCO }}$. Although we know the relevance of the spin state of $\mathrm{Fe}^{2+}$ in $(\mathrm{Mg}, \mathrm{Fe}) \mathrm{O}$, the second most abundant phase in lower mantle, on the radiative conductivity ${ }^{5,6}$ or that highspin $\mathrm{Fe}^{3+}(S=5 / 2)$ transits to the low-spin state $(S=1 / 2)$ in
$\mathrm{Fe}_{2} \mathrm{O}_{3}$ at pressures around $50 \mathrm{GPa},{ }^{7,8}$ the problem of predicting spin-crossover pressures in materials science is still a challenge. A comprehensive characterization of high-spin to low-spin (or intermediate spin) ${ }^{1}$ phenomena requires knowledge of the electronic structure of materials, a major problem because optical absorption measurements in extreme conditions are tricky. ${ }^{6}$ Besides, the influence of the particular $d^{n}$ configuration and site symmetry on the spin state must also be considered. Depending on whether the ground state associated with $e$ electrons is orbitally degenerate, the crystal environment will be unstable under low-symmetry distortions (Jahn-Teller effect), yielding free-energy reduction. ${ }^{9-11}$ As a result, the interplay between the Jahn-Teller distortion and the spin state strongly affects both the magnetic moment and local structure of the transition-metal ion, and thus the associated physical properties of the material. Perovskitetype $\mathrm{Mn}^{3+}$ oxides and fluorides exhibit a great variety of structures illustrating this effect. ${ }^{9,10}$ The occurrence of given properties is driven by strong correlation effects associated with electron-electron interactions, the Jahn-Teller effect, spin state and tilting of $\mathrm{Mn}^{3+}$ octahedra. Such effects are coupled to the lattice, and thus can be modified by external pressure. Magnetic transformation from ferromagnetic to antiferromagnetic in $\mathrm{CsMnF}_{4}$ (Refs. 11-13), metal to insulator transition phenomena in $\mathrm{LaMnO}_{3}$ (Ref. 14), or colossal magnetoresistance and spintronics in $\mathrm{Sm}_{0.55} \mathrm{Sr}_{0.45} \mathrm{MnO}_{3}$ or $\mathrm{La}_{2-2 x} \mathrm{Sr}_{1+2 x} \mathrm{Mn}_{2} \mathrm{O}_{7}$ (Refs. 15 and 16) are examples, for which reason they have received considerable attention. Our interest in fluorides stems from their advantage over oxides when obtaining the electronic structure in extreme conditions. In particular, the Jahn-Teller distortion, its associated electronic structure and stabilization energy $\left(E_{\mathrm{JT}}\right)$, the spin state, and orbital ordering of $\mathrm{Mn}^{3+}$ ions can be jointly studied by optical absorption spectroscopy under high-pressure conditions. ${ }^{11,12,17}$ 

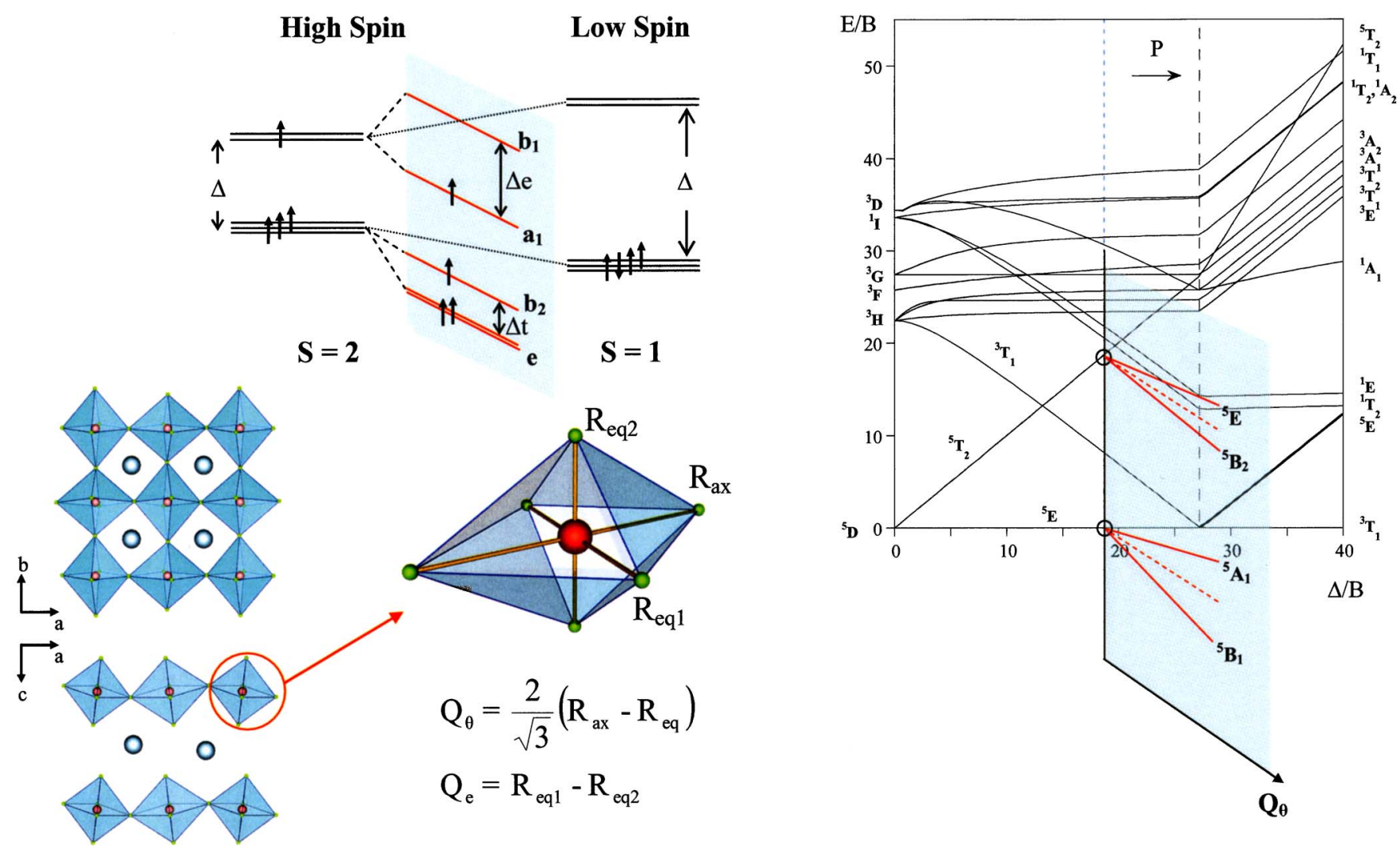

FIG. 1. (Color online) (Left) Diagram of the $d$ levels of $\mathrm{Mn}^{3+}$ in octahedral $(O)$ and elongated tetragonal $\left(D_{4}\right)$ coordinations, showing the $E \otimes e$ Jahn-Teller high-spin and $O$ low-spin configurations. (Right) Variation of the state energies (Tanabe-Sugano diagram) for $3 d^{4}$ ions calculated for $C / B=4.6$ as a function of the crystal-field energy in terms of the Racah parameter $B$ (Ref. 11). Some states have been omitted in the diagram for the sake of clarity. The ${ }^{5} E \leftrightarrow{ }^{3} T_{1}$ crossover crystal field $(\Delta / B)_{\mathrm{SCO}}$ is $27 ; B \approx 0.1 \mathrm{eV}$ for Mn ${ }^{3+}$ in fluorides and oxides $($ Refs. 2-4). The splittings of the ${ }^{5} E$ and ${ }^{5} T_{2}$ states due to the Jahn-Teller effect are given as a function of the normal coordinate $Q_{\theta}$, keeping a ratio $\Delta_{e} / \Delta_{t}=3.7$ (Ref. 11). The crystal structure of the layered perovskite $\mathrm{CsMnF}_{4}$ (space group: $P 4 / n$ ), showing the in-layer and intralayer views, together with the elongated $\left(\mathrm{MnF}_{6}\right)^{3-}$ complex, with axial and equatorial Mn-F distances, $R_{\mathrm{ax}}, R_{\text {eq1 }}$ and $R_{\text {eq2 }}$, are given bottom left. The normal coordinates, $Q_{\theta}$ and $Q_{\varepsilon}$, representing the tetragonal and rhombic distortions, respectively, are given as a function of the three Mn-F distances. $R_{\text {eq }}=1 / 2\left(R_{\text {eq } 1}+R_{\text {eq2 }}\right)$. Note the antiferrodistortive structure shown by the $\left(\mathrm{MnF}_{6}\right)^{3-}$ octahedra in the $a, b$ layer.

\section{EXPERIMENT}

Single crystals of $\mathrm{CsMnF}_{4}$ were obtained from dehydration of the $\mathrm{CsMnF}_{4} \cdot\left(\mathrm{H}_{2} \mathrm{O}\right)$; its tetragonal $P 4 / n$ crystal structure was checked by $\mathrm{x}$-ray diffraction with lattice parameters of $a=7.947(3) \AA$ and $c=6.340(3) \AA$. A Merril-Basset diamond anvil cell, Diamond Optics, Inc., was employed for high-pressure optical absorption spectroscopy. Every experiment was done loading a suitable single-crystal platelet $\left(150 \times 100 \times 30 \mu \mathrm{m}^{3}\right)$ of $\mathrm{CsMnF}_{4}$ in the diamond anvil cell with several ruby chips for pressure calibration. DowCorning 200 silicone oil was employed as pressuretransmitter medium, as it provides a suitable operation for optical spectroscopy at high pressures $(P>20 \mathrm{GPa})$. In order to estimate contributions from non hydrostatic components, we have measured the variation of the Ruby linewidth as a function of pressure in several Ruby chips. The R1 linewidth of $0.6 \mathrm{~nm}$ is almost constant in the range $0-20 \mathrm{GPa}$, and increases continuously from 0.6 to about $1.0 \mathrm{~nm}$ in the 20-46 GPa range. The high-pressure spectroscopy setup has been described elsewhere. ${ }^{17}$ The absorption spectra were taken with the light propagating perpendicular to the $c$ lay- ers, and are nearly isotropic as corresponds to antiferrodistortive layers of $\left(\mathrm{MnF}_{6}\right)^{3-}$ even in the monoclinic highpressure phase.

\section{RESULTS AND DISCUSSION}

\section{A. Structural correlations in $\left(\mathrm{MnF}_{6}\right)^{3-}$ systems: Pressure effects on $\mathrm{CsMnF}_{4}$}

Here, we investigate the interplay between the Jahn-Teller effect, spin state, and tilting of $\left(\mathrm{MnF}_{6}\right)^{3-}$ octahedra in the layer perovskite $\mathrm{CsMnF}_{4}$ as a function of pressure. The aim is to elucidate whether pressure induces tilts of the $\left(\mathrm{MnF}_{6}\right)^{3-}$ octahedra preserving the Jahn-Teller distortion and high spin, as occurs along the $A \mathrm{MnF}_{4}$ series upon volume reduction, ${ }^{11,13}$ or whether it mainly reduces their axial distortion toward $\mathrm{O}$ symmetry, the latter modification favoring low-spin stabilization. The electronic spectrum of $\mathrm{CsMnF}_{4}$ and its variation with pressure in the $0-46 \mathrm{GPa}$ range are shown in Fig. 2. At ambient pressure, it consists of three broadbands, $E_{1}=1.89 \mathrm{eV}, E_{2}=2.26 \mathrm{eV}$, and $E_{3}=2.79 \mathrm{eV}$, corresponding to interelectronic transitions within $d^{4}$ from 


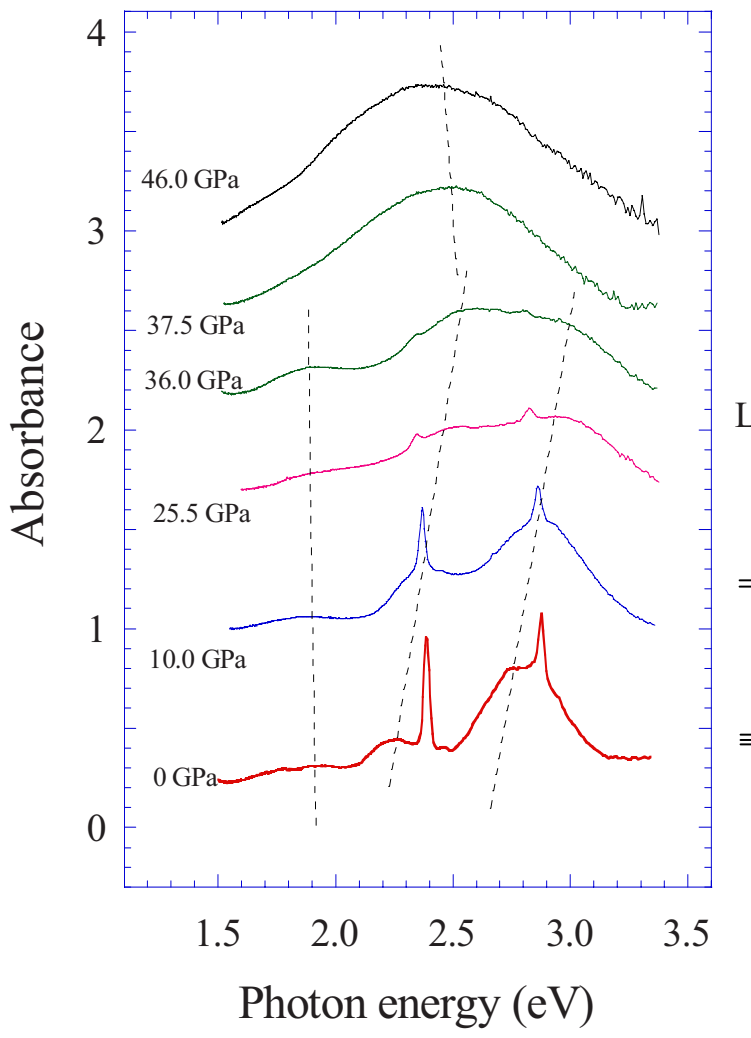

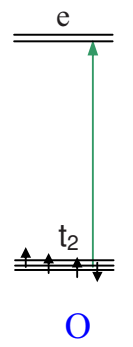

Low spin, $S=1$
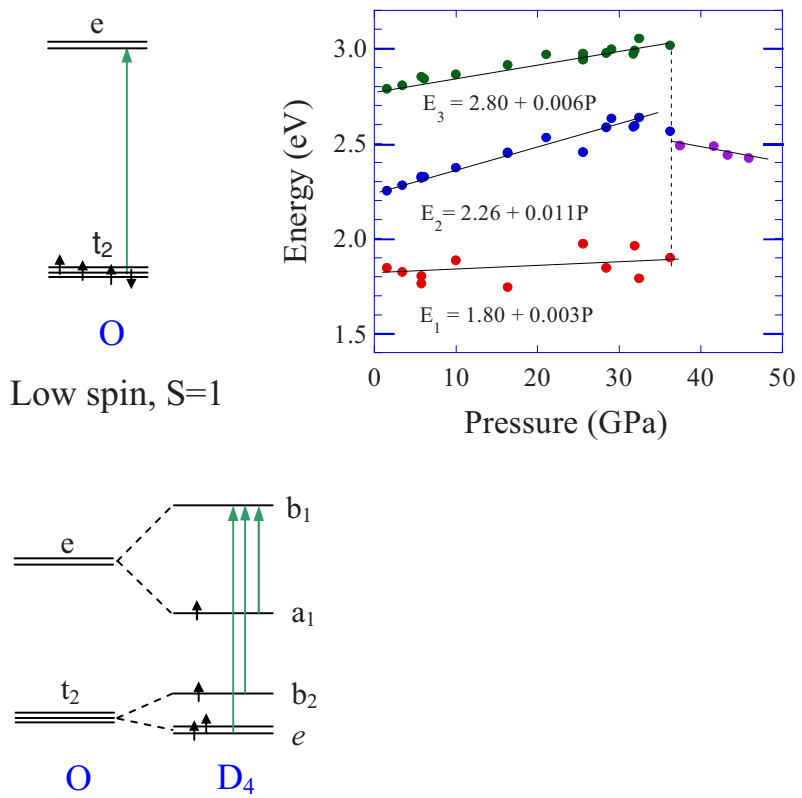

High spin, $\mathrm{S}=2$

FIG. 2. (Color online) Variation of the optical absorption spectrum of single crystal of $\mathrm{CsMnF}_{4}$ with pressure in the $0-46 \mathrm{GPa}$ range at room temperature (upstroke). Broken lines illustrate the pressure-induced shift for the three broadbands. Note the abrupt change of the absorption spectrum at $37 \mathrm{GPa}$. The energy level diagram for $\mathrm{Mn}^{3+}$ in elongated- $D_{4}$ and $O$ symmetries with corresponding crystal-field excitations are indicated on the right. The variation of $E_{1}, E_{2}$, and $E_{3}$ as a function of pressure is given top right. Estimated energy errors are $10 \mathrm{meV}$. Note that the Jahn-Teller-related broadband structure and the two spin-flip peaks are observed up to 36 GPa, but both undergo abrupt jumps at the critical pressure, $P_{C}=37 \mathrm{GPa}$.

the ${ }^{5} B_{1}$ ground state to the ${ }^{5} A_{1},{ }^{5} B_{2}$, and ${ }^{5} E$ excited states, respectively, according to the energy level diagram of Figs. $1-3$. Their energy provides the $d$-splitting pattern due to the $D_{4}$-elongated Jahn-Teller distortion of $\left(\mathrm{MnF}_{6}\right)^{3-}$. Note that in $\mathrm{O}$, the spectrum would consist of a single broadband $\left({ }^{5} E\right.$ $\rightarrow{ }^{5} T_{2}$ ), whose energy is $\Delta .{ }^{11}$ On the basis of correlations established elsewhere, ${ }^{11,17}$ the three transition energies in $D_{4}$ (four in $D_{2}$ ) are related to the equatorial Mn-F distance, $R_{\text {eq }}$, and the tetragonal and rhombic normal coordinates, $Q_{\theta}$ and $Q_{\varepsilon}$, describing the low-symmetry distortion of the $\left(\mathrm{MnF}_{6}\right)^{3-}$ octahedra (Fig. 1). In fact, $E_{1}, E_{2}$, and $E_{3}$ provide the socalled equatorial crystal-field parameter, $\Delta(\mathrm{eq})=E_{2}$, and the tetragonal splitting of the parent octahedral $e$ and $t_{2}$ orbitals, $\Delta_{e}=E_{1}=4 E_{\mathrm{JT}}$, and $\Delta_{t}=E_{3}-E_{2}$ (Ref. 11). The narrow peaks, $E_{\mathrm{SP} 1}=2.39 \mathrm{eV}$ and $E_{\mathrm{SP} 2}=2.88 \mathrm{eV}$, correspond to spin-flip transitions: ${ }^{5} E \rightarrow{ }^{3} E$ and ${ }^{5} E \rightarrow{ }^{3} A_{2}$ in $\mathrm{O}$ (both ${ }^{5} B_{1} \rightarrow{ }^{3} B_{1}$ in $D_{4}$ ), the transition mechanism of which is activated by the Mn-F-Mn exchange interaction, ${ }^{12,17}$ similar to the $\mathrm{K}_{2} \mathrm{CrCl}_{4}$ ferromagnet involving the isoelectronic $\mathrm{Cr}^{2+}$ (Ref. 18). Their intensity decreases with the Mn-F-Mn bending angle as it is confirmed through the variation of the optical spectrum along the series $A \mathrm{MnF}_{4} ; A: \mathrm{Cs} \rightarrow \mathrm{Tl} \rightarrow \mathrm{Na}$ (Fig. 3), and thus can probe tilting phenomena. The spectra of Fig. 3 provide a direct correlation between the transition oscillator strength (proportional to the peak area) and the Mn-F-Mn tilting angle $\varphi$, indicating that the electric-dipole mechanism is likely to rely on the exchange interaction via the $\varphi$-dependent $e-t$ and $e$ - $e$-coupling exchange [GoodenoughKanamori-Anderson (G-K-A) rules]. ${ }^{19-21}$ The dependence of the spin-flip intensity with $\varphi$ as $-0.9+2.1 \cos ^{2}(\varphi) \approx \cos (2 \varphi)$ supports this view (Fig. 3). Note, however, that the exchange mechanism is weaker in the axially $\mathrm{F}$-sharing linear chains of $\left(\mathrm{MnF}_{6}\right)^{3-}$ units $\left(A \mathrm{MnF}_{5}\right.$ series $)$ or in $\left(\mathrm{MnF}_{6}\right)^{3-}$ isolated systems $\left(A_{3} \mathrm{MnF}_{6}\right)$, and, consequently, the spin-flip peaks are missed in their corresponding $\mathrm{O} A$ spectra (Fig. 4). Hence, spin-flip transitions appear as efficient probes for exploring tilting phenomena in $\mathrm{CsMnF}_{4}$, as already suggested in previous pressure studies in $\mathrm{NaMnF}_{4}$ (Ref. 17). Although departures of $\varphi$ from $180^{\circ}$ in $A \mathrm{MnF}_{4}(A: \mathrm{Cs} \rightarrow \mathrm{Tl} \rightarrow \mathrm{Na})$ reduce the spin-flip intensity, the $E_{1}, E_{2}$, and $E_{3}$ energies are similar according to the same local structure of $\left(\mathrm{MnF}_{6}\right)^{3-}$ (Ref. 11). Nevertheless, a substantial variation in these energies is attained if the coordination geometry around $\mathrm{Mn}^{3+}$ is modified by crystal anisotropy, as occurs in $\mathrm{K}_{3} \mathrm{MnF}_{6}, \mathrm{Tl}_{2} \mathrm{MnF}_{5} \cdot \mathrm{H}_{2} \mathrm{O}$, and $\mathrm{CsMnF}_{4}$, where $\left(\mathrm{MnF}_{6}\right)^{3-}$ units appear independent, in chains, and forming layers, respectively (Fig. 4). The JahnTeller splitting obtained from the absorption spectra, $\Delta_{e}$ and $\Delta_{t}$, both depend linearly on $Q_{\theta}$ with $\Delta_{e} / \Delta_{t}=3.7$. This noteworthy result indicates that the Jahn-Teller electron-ion coupling $E \otimes e$ is four times bigger than the $T \otimes e$ coupling, 

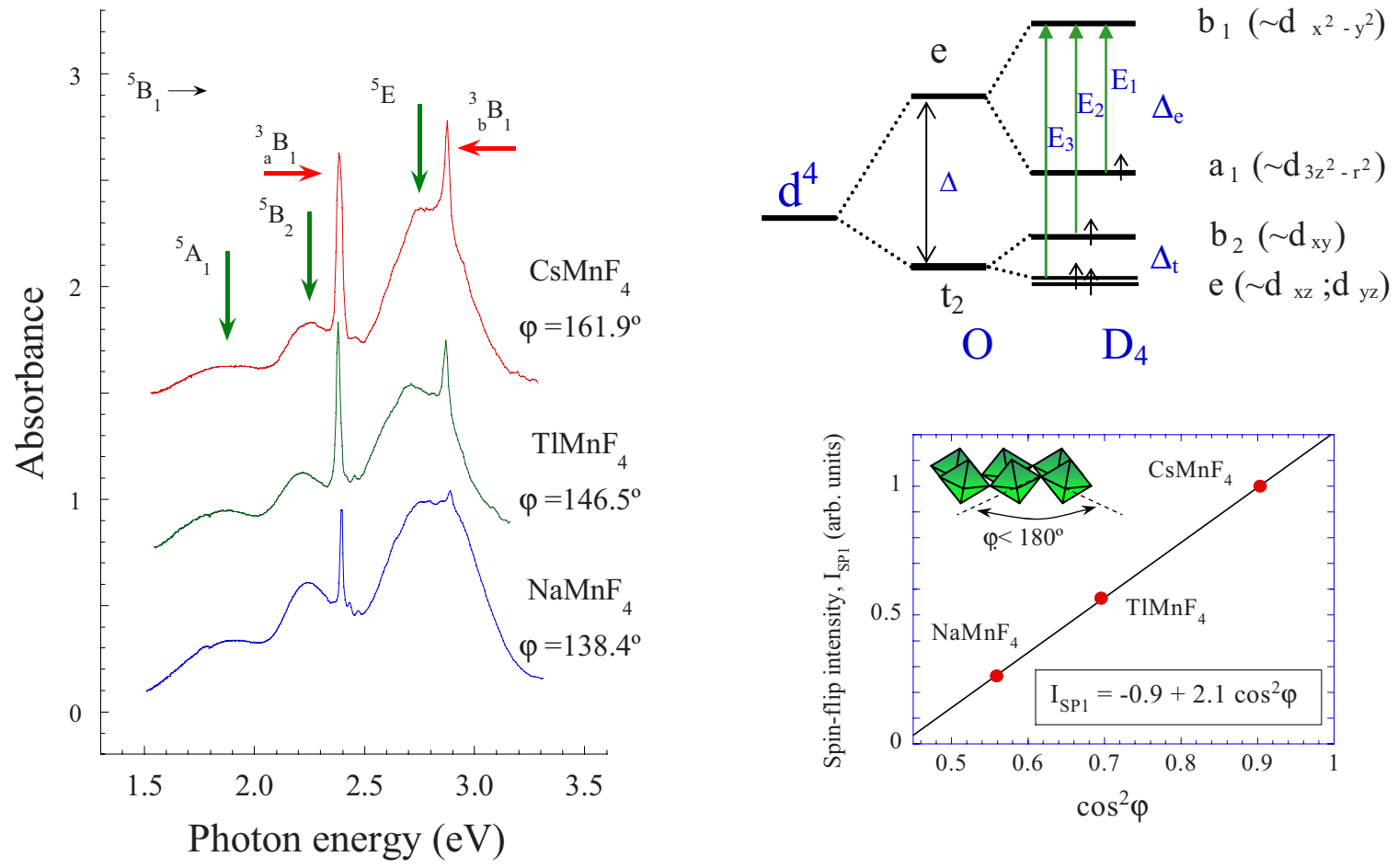

FIG. 3. (Color online) Optical absorption spectra of $\mathrm{NaMnF}_{4}, \mathrm{TlMnF}_{4}$, and $\mathrm{CsMnF}_{4}$ single crystals. $R_{\mathrm{ax}}$ and $R_{\mathrm{eq}}$ are $2.15,1.82 \AA$ for $\mathrm{TlMnF}_{4}$ and 2.17, $1.84 \AA$ for $\mathrm{NaMnF}_{4}$ and $\mathrm{CsMnF}_{4}$. The Mn-F-Mn bond angle is indicated on the right. ${ }^{13}$ The three spin-allowed crystalfield transitions, $E_{1}, E_{2}$, and $E_{3}$, and the spin-flip peaks, $E_{\mathrm{SP} 1}$ and $E_{\mathrm{SP} 2}$, are indicated by vertical and horizontal arrows, respectively. The spin-flip integrated peak intensity decreases with the tilting angle, $\theta=180-\varphi, \varphi$ being the Mn-F-Mn bond angle. Its variation is linear with $\cos ^{2} \varphi=\cos ^{2} \theta$, showing the exchange-induced electric-dipole mechanism of the spin-flip transitions (Refs. 12, 17, and 18). The errors are 0.05 and 0.005 for the relative intensity and $\cos ^{2} \varphi$, respectively.

which explains the greater axial distortions exhibited by Jahn-Teller systems involving $e$ electrons instead of $t_{2}$. Thus, $E_{1}, E_{2}$, and $E_{3}$ together with spin-flip peaks provide a useful probe for exploring electronic properties and hence the JahnTeller effect of $\mathrm{Mn}^{3+}$ in pressure experiments.

\section{B. Spin transition and Jahn-Teller suppression in $\mathrm{CsMnF}_{4}$}

Two relevant features are observed in the evolution of the $\mathrm{CsMnF}_{4}$ spectrum with pressure in Fig. 2: first, the JahnTeller-related triple-broadband structure is observed up to $36 \mathrm{GPa}$, and second, the ${ }^{5} B_{1} \rightarrow{ }^{3} B_{1}$ spin-flip peaks decrease from ambient pressure to around $36 \mathrm{GPa}$. Both features indicate that pressure mainly induces $\left(\mathrm{MnF}_{6}\right)^{3-}$ tilts below $37 \mathrm{GPa}$, still preserving the Jahn-Teller distortion. Above $37 \mathrm{GPa}$, the triple-broadband structure sharply transforms into a single broadband located at $2.5 \mathrm{eV}$, whereas spin-flip peaks completely disappear. The abrupt transition at $37 \mathrm{GPa}$ involves a marked piezochromism with a color change of the crystal from light brown to pinkish red. We associate this change with the simultaneous suppression of the Jahn-Teller distortion and the spin transition $(S=2 \rightarrow 1)$ in $\mathrm{Mn}^{3+}$. The collapse of the low-symmetry $3 \mathrm{~d}$-splitting pattern into one single electronic band, which is basically related to the $t_{2}$ $\rightarrow e$ one-electron transition as expected in $\mathrm{O}$, supports the suppression of the Jahn-Teller distortion (Fig. 2). The band energy at $40 \mathrm{GPa}$ corresponds to $\Delta=2.5 \mathrm{eV}$, and, as we dem- onstrate below, is in agreement with the proposed model.

Upon pressure release, a reversible abrupt change occurs at about $30 \mathrm{GPa}$, yielding the high-spin phase. Both the large hysteresis $(>5 \mathrm{GPa})$ and observed piezochromism indicate that the simultaneous Jahn-Teller suppression and spin change likely involve a first-order structural-phase transition in $\mathrm{CsMnF}_{4}$. However, it must be pointed out that although the pressure hysteresis can be affected by the pressuretransmitter medium, we have verified that the Ruby lines show no hysteresis effect in the same pressure range. Therefore, under the assumption that there is no residual strain in the crystal left on the pressure release, the high-spin to lowspin transition must be fully associated with a first-order phase transition in $\mathrm{CsMnF}_{4}$.

\section{Interplay between spin transition and Jahn-Teller effect: Crystal-field model}

In contrast to $\mathrm{Ni}^{3+}\left(t_{2}^{6} e^{1} ; S=1 / 2\right)$ and $\mathrm{Co}^{3+}\left(t_{2}^{6} e^{0} ; S=0\right)$, with a strong tendency to form a low-spin ground state, $1,22-29$ $\mathrm{Mn}^{3+}$ in fluorides and oxides exhibits a high-spin ground state $\left(t_{2}^{3} e^{1} ; S=2\right)$ in spite of $\Delta \approx 2 \mathrm{eV}$ being similar for all these ions. ${ }^{3,4,29}$ Within $\mathrm{O}$, the spin crossover for $\mathrm{Mn}^{3+}$ $\left[t_{2}^{3} e^{1}\left({ }^{5} E\right) \leftrightarrow t_{2}^{4} e^{0}\left({ }^{3} T_{1}\right)\right]$ and $\mathrm{Ni}^{3+}\left[t_{2}^{5} e^{2}\left({ }^{4} T_{1}\right) \leftrightarrow t_{2}^{6} e^{1}\left({ }^{2} E\right)\right]$ takes place typically at $\Delta_{\mathrm{SCO}} \approx 2.7$ and $2.1 \mathrm{eV}$, respectively. ${ }^{3,4,30}$ Actually, spin crossover occurs if the associated free energy of the high-spin state becomes greater than that of the low- 
a)

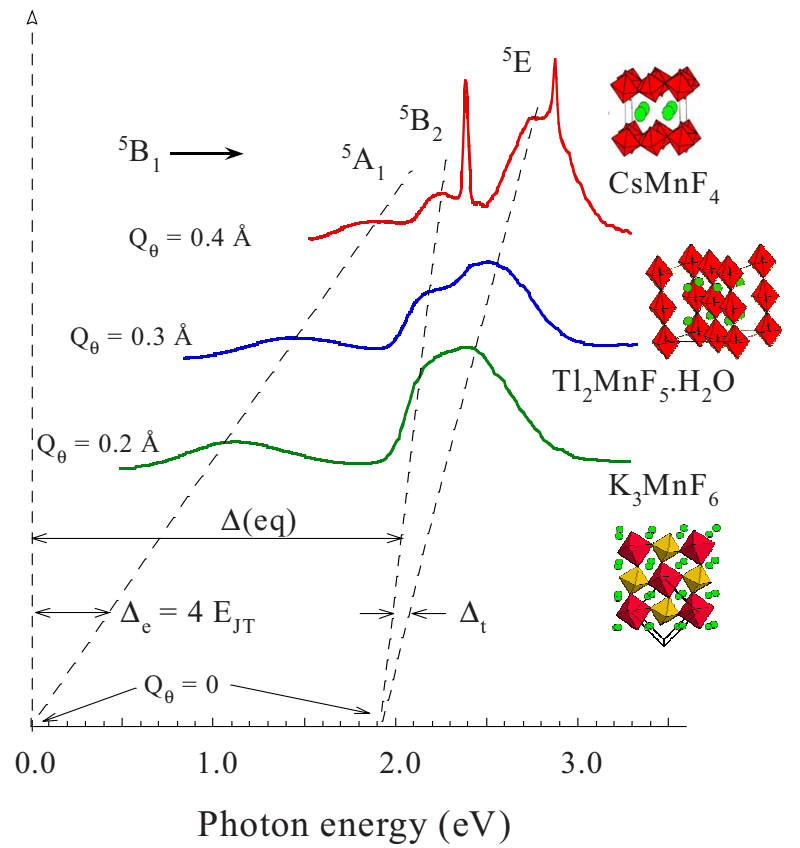

b)

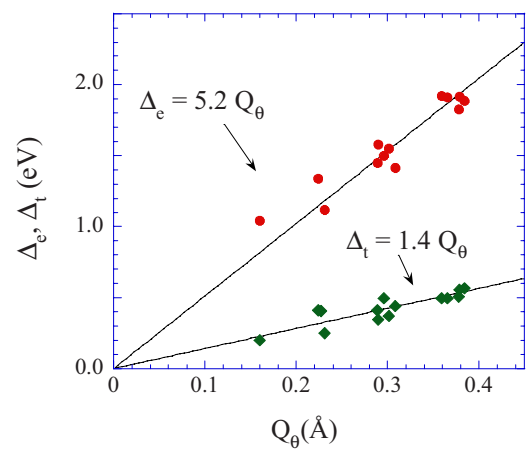

FIG. 4. (Color online) (a) Optical absorption spectra of $\mathrm{CsMnF}_{4}, \mathrm{Tl}_{2} \mathrm{MnF}_{5} \cdot \mathrm{H}_{2} \mathrm{O}$, and $\mathrm{K}_{3} \mathrm{MnF}_{6}$ single crystals. Their crystal structure, showing different crystal dimensionality, is shown on the right. Values of Mn-F distances and normal coordinates, $R_{\mathrm{ax}}$ and $R_{\mathrm{eq}}\left(Q_{\theta}\right.$ and $\left.Q_{\varepsilon}\right)$, respectively, are 2.06, $1.86 \AA(0.23,0.04 \AA)$ for $\mathrm{K}_{3} \mathrm{MnF}_{6}, 2.08,1.83 \AA(0.29,0.03 \AA)$ for $\mathrm{Tl}_{2} \mathrm{MnF}_{5}$, and $2.17,1.84 \AA(0.38,0.04 \AA)$ for $\mathrm{CsMnF}_{4}$ (Ref. 11). Note that $E_{1}, E_{2}$, and $E_{3}$ shift to higher energies with $Q_{\theta}$. (b) Correlation between the tetragonal splitting, $\Delta_{e}$ and $\Delta_{t}$, and the normal coordinate, $Q_{\theta}$, for several $\mathrm{Mn}^{3+}$ fluorides (Ref. 11) showing an almost linear dependence: $\Delta_{e}=5.2 Q_{\theta}$ and $\Delta_{t}=1.4 Q_{\theta}$ (in eV and $\AA$ units, respectively).

spin state. Therefore, the effective energy for spin crossover $\Delta_{\mathrm{SCO}}$ is thus released by the lattice-relaxation energy $E_{\mathrm{LR}}$, due to the electron-lattice coupling between low-spin and high-spin states in $\mathrm{O}$. Interestingly, $E_{\mathrm{LR}}$ can be obtained from optical spectroscopy and is given approximately by $S \hbar \omega$ $\approx 0.1-0.3 \mathrm{eV}$ for trivalent transition-metal ions..$^{3,4,12}$ Additionally, this energy can be either reduced or increased depending on whether the high-spin or low-spin states exhibit a strong Jahn-Teller effect associated with $e$ electrons $(E \otimes e)$. Within the $d^{4}$ high-spin configuration $\left(t_{2}^{3} e^{1}\right)$, the lone $e$ electron is responsible for the strong axial distortion exhibited by the $\left(\mathrm{MnF}_{6}\right)^{3-}$ octahedra, ${ }^{11}$ whose electronic structure is sketched in Fig. 1. As a result, the energy separation between the $e$ electron $\left(a_{1}\right)$ and $t_{2}$ electrons $\left(b_{2}+e\right)$, which is $\Delta$ in $\mathrm{O}$, reduces approximately by $E_{1} / 2=0.9 \mathrm{eV}$ (Ref. 11) in $D_{4}$, yielding high-spin stabilization. This gain of electronic energy is, nevertheless, accompanied by an increase in elastic energy associated with the axial distortion. According to $E$ $\otimes e$ theory, ${ }^{11,17}$ the energy gain, $E_{\mathrm{JT}}$, is given by $E_{1} / 4$ $=0.45 \mathrm{eV} / \mathrm{Mn}^{3+}$ in $A \mathrm{MnF}_{4}$ (Fig. 3). An opposite situation is attained for $\mathrm{Ni}^{3+}$ since the low-spin state has one electron in the $e$ orbitals, and thus exhibits a strong Jahn-Teller effect in contrast to its high-spin state. Consequently, $\Delta_{\mathrm{SCO}}$ for $\mathrm{Ni}^{3+}$ is smaller than that for $\mathrm{Mn}^{3+}$ to encompass spin transition. Therefore, the effective $\Delta_{\mathrm{SCO}}$ increases or decreases by $E_{\mathrm{JT}}$ depending on whether we are dealing with $\mathrm{Mn}^{3+}$ or $\mathrm{Ni}^{3+}$, respectively. In other words, the energy balance between low-spin and high-spin states is given by

$$
\begin{aligned}
& E\left({ }^{5} E\right)-E_{\mathrm{JT}}=E\left({ }^{3} T_{1}\right)-E_{\mathrm{LR}} \text { for } \mathrm{Mn}^{3+}, \\
& E\left({ }^{4} T_{1}\right)=E\left({ }^{2} E\right)-E_{\mathrm{JT}}-E_{\mathrm{LR}} \text { for } \mathrm{Ni}^{3+},
\end{aligned}
$$

or likewise,

$$
\begin{gathered}
\Delta_{\mathrm{SCO}}\left[\mathrm{Mn}^{3+}\right] \approx U+E_{\mathrm{JT}}-E_{\mathrm{LR}}, \\
\Delta_{\mathrm{SCO}}\left[\mathrm{Ni}^{3+}\right] \approx U-E_{\mathrm{JT}}-E_{\mathrm{LR}},
\end{gathered}
$$

where $U=2.7$ and $2.1 \mathrm{eV}$ for $\mathrm{Mn}^{3+}$ and $\mathrm{Ni}^{3+}$, respectively. Taking $E_{\mathrm{JT}}=0.4 \mathrm{eV}$ and $E_{\mathrm{LR}}=0.2 \mathrm{eV}$ for both ions, we obtain $\Delta_{\mathrm{SCO}}=2.9$ and $1.5 \mathrm{eV}$, respectively, which explains why the Jahn-Teller effect stabilizes $\mathrm{Mn}^{3+}$ high-spin and $\mathrm{Ni}^{3+}$ lowspin at ambient pressure. In fact, $\mathrm{Ni}^{3+}$ is low spin $\left({ }^{2} E\right)$ in $R \mathrm{NiO}_{3}$ perovskites ( $R$ : rare earth), ${ }^{24,25} \mathrm{Ni}^{3+}$-doped $\mathrm{LaAlO}_{3}$ (Ref. 22), and $\mathrm{CsCaNiF}_{6}$ (Ref. 30), whereas corresponding $\mathrm{Mn}^{3+}$ compounds are high spin at ambient conditions. The present model foresees that in the eventual case of JahnTeller suppression by pressure $\left(E_{\mathrm{JT}}=0\right)$, the spin crossover in $\mathrm{CsMnF}_{4}$ would occur at $\Delta_{\mathrm{SCO}}=2.5 \mathrm{eV}$ as is observed in Fig. 2.

\section{CONCLUSIONS}

Our observations demonstrate that the pressure-induced Jahn-Teller suppression leads to a sharp spin crossover in $\mathrm{CsMnF}_{4}$ taking place at $37 \mathrm{GPa}$. The spin transition occurs if 
the high-spin ${ }^{5} E$ free energy surpasses the low-spin ${ }^{3} T_{1}$ free energy, which is attained for $\Delta_{\mathrm{SCO}} \approx 2.5 \mathrm{eV}$, once the axial distortion is suppressed $\left(E_{\mathrm{JT}}=0\right)$. The low-spin phase is stable at least to $46 \mathrm{GPa}$, the highest pressure applied in the present studies. Upon pressure release, a reversible abrupt change occurs at about $30 \mathrm{GPa}$, yielding the high-spin phase. Both the large hysteresis ( $>5 \mathrm{GPa}$ ) and observed piezochromism indicate that the simultaneous Jahn-Teller suppression and spin change involve a first-order structural-phase transition in $\mathrm{CsMnF}_{4}$. These results are noteworthy since they go a long way to explaining some essential features related to the spin state of transition-metal ions in oxides and fluorides, and have to be considered when discussing the cooperative Jahn-Teller effect in complex systems where electron delocalization or high-pressure conditions play a key role.

\section{ACKNOWLEDGMENTS}

This work was financially supported by the Spanish MEyC (Project Reference No. MAT2005-00099). F.R. acknowledges partial support from the I3 Intensification Research Program of the Autonomous Government and the University of Cantabria.
*Corresponding author. rodriguf@unican.es

${ }^{1}$ P. Gütlich and H. A. Goodwin, Spin Crossover in Transition Metal Compounds II (Springer, Heidelberg, 2004).

${ }^{2}$ J. S. Griffith, The Theory of Transition Metal Ions (Cambridge University Press, Cambridge, 1980).

${ }^{3}$ A. B. P. Lever, Inorganic Electronic Spectroscopy: Studies in Physical and Theoretical Chemistry (Elsevier, New York, 1984).

${ }^{4}$ N. S. Hush and R. J. M. Hobbs, Prog. Inorg. Chem. 10, 259 (1968).

${ }^{5}$ J. F. Lin, V. V. Struzhkin, S. D. Jacobsen, M. Y. Hu, P. Chow, J. Kung, H. Z. Liu, H. K. Mao, and R. J. Hemley, Nature (London) 436, 377 (2005).

${ }^{6}$ A. F. Goncharov, V. V. Struzhkin, and S. D. Jacobsen, Science 312, 1205 (2006).

${ }^{7}$ M. P. Pasternak, G. K. Rozenberg, G. Y. Machavariani, O. Naaman, R. D. Taylor, and R. Jeanloz, Phys. Rev. Lett. 82, 4663 (1999).

${ }^{8}$ J. Badro, G. Fiquet, V. V. Struzhkin, M. Somayazulu, H. K. Mao, G. Shen, and T. Le Bihan, Phys. Rev. Lett. 89, 205504 (2002).

${ }^{9}$ J. B. Goodenough, Rep. Prog. Phys. 67, 1915 (2004).

${ }^{10}$ M. Imada, A. Fujimori, and Y. Tokura, Rev. Mod. Phys. 70, 1039 (1988), and references therein.

${ }^{11}$ F. Rodriguez and F. Aguado, J. Chem. Phys. 118, 10867 (2003).

${ }^{12}$ F. Rodriguez, P. Núñez, and M. Marco de Lucas, J. Solid State Chem. 110, 370 (1994).

${ }^{13}$ M. Ishizuka, S. Henmi, S. Endo, M. C. Moron, and F. Palacio, J. Magn. Magn. Mater. 196-197, 440 (1999), and references therein.

${ }^{14}$ I. Loa, P. Adler, A. Grzechnik, K. Syassen, U. Schwarz, M. Hanfland, G. Kh. Rozenberg, P. Gorodetsky, and M. P. Pasternak, Phys. Rev. Lett. 87, 125501 (2001).
${ }^{15}$ J. M. De Teresa, M. R. Ibarra, P. Algarabel, L. Morellon, B. Garcia-Landa, C. Marquina, C. Ritter, A. Maignan, C. Martin, B. Raveau, A. Kurbakov, and V. Trounov, Phys. Rev. B 65, 100403(R) (2002).

${ }^{16}$ H. M. Ronnow, Ch. Renner, G. Aeppli, T. Kimura, and Y. Tokura, Nature (London) 440, 1025 (2006).

${ }^{17}$ F. Aguado, F. Rodriguez, and P. Núñez, Phys. Rev. B 67, 205101 (2003).

${ }^{18}$ P. Day, A. K. Gregson, and D. H. Leech, Phys. Rev. Lett. 30, 19 (1973).

${ }^{19}$ J. B. Goodenough, Phys. Rev. 100, 564 (1955).

${ }^{20}$ J. Kanamori, J. Phys. Chem. Solids 10, 87 (1959).

${ }^{21}$ P. W. Anderson, Phys. Rev. 115, 2 (1959).

${ }^{22}$ T. A. Ivanova, V. E. Petrashen, N. V. Chezhina, and Y. V. Yablokov, Phys. Solid State 44, 1468 (2002).

${ }^{23}$ R. Stoyanova, E. Zhecheva, R. Alcantara, and J. L. Tirado, J. Phys. Chem. B 108, 4053 (2004).

${ }^{24}$ J. A. Alonso, J. L. Garcia-Munoz, M. T. Fernandez-Diaz, M. A. G. Aranda, M. J. Martinez-Lope, and M. T. Casais, Phys. Rev. Lett. 82, 3871 (1999).

${ }^{25}$ C. Piamonteze, F. M. F. de Groot, H. C. N. Tolentino, A. Y. Ramos, N. E. Massa, J. A. Alonso, and M. J. Martinez-Lope, Phys. Rev. B 71, 020406(R) (2005).

${ }^{26}$ J. Q. Yan, J. S. Zhou, and J. B. Goodenough, Phys. Rev. B 69, 134409 (2004).

${ }^{27}$ L. G. Vanquickenborne, K. Pierloot, and E. Duyvejonck, Chem. Phys. Lett. 224, 207 (1994).

${ }^{28}$ F. A. Cotton and M. D. Meyers, J. Am. Chem. Soc. 82, 5023 (1960).

${ }^{29}$ E. Alter and R. Hoppe, Z. Anorg. Allg. Chem. 405, 167 (2004).

${ }^{30}$ D. Oelkrug, Struct. Bonding (Berlin) 9, 1 (1971). 